\begin{tabular}{c|c|c}
\hline ISSN 2525-4812 (versão online) & Recebido em: 2/4/2019 & Revista Terceira \\
ISSN 2238-7641 (versão impressa) \\
$\begin{array}{c}\text { http://www.revistaterceiramargem.com/ } \\
\text { index.php/terceiramargem/index }\end{array}$ & $\begin{array}{c}\text { Aprovado em: 20/1/2020 } \\
\text { Período de publicação: jan., 2021 }\end{array}$ & $\begin{array}{c}\text { Margem Amazônia } \\
\text { (v. 6 • n. especial 16 • Jan. 2021) }\end{array}$ \\
\hline
\end{tabular}

Como citar o artigo:

PRADO, R. B. Serviços ecossistêmicos: estado atual e desafios para a pesquisa na Amazônia. Revista Terceira Margem Amazônia. v. 6, n. especial 16, p. 11-22, 2021. DOI: http://dx.doi.org/10.36882/2525-4812.2021v6i16.ed.esp.p11-22

\title{
SERVIÇOS ECOSSISTÊMICOS: ESTADO ATUAL E DESAFIOS PARAA PESQUISA NA AMAZÔNIA
}

Rachel Bardy Prado ${ }^{1}$

\begin{abstract}
Resumo: Os serviços ecossistêmicos (SEs) têm sido compreendidos como os benefícios que o ser humano obtém dos ecossistemas. Portanto, esses serviços recebem influência direta e indireta das ações antrópicas, que nas últimas décadas têm exercido forte pressão sobre os ecossistemas, colocando-os sob ameaça de redução ou extinção. Alguns conceitos, avanços e vantagens da abordagem de SE são apresentados nesta publicação. O bioma Amazônia é muito abundante em biodiversidade e na prestação de diversos SEs com reflexos em diferentes escalas, mas também muitas são as ameaças que sofrem, sendo aqui de igual forma elencadas. Por fim é nesse contexto ambiental e socioeconômico complexo, diverso e sempre muito visível aos espectadores nacionais e internacionais que se apresentam os grandes desafios da pesquisa com foco na sustentabilidade e provisão dos serviços ecossistêmicos, sendo apresentados e discutidos ao longo deste texto.
\end{abstract}

Palavras-chave: ferramentas para suporte à decisão, PSA, biodiversidade.

\section{ECOSYSTEM SERVICES: CONTEMPORARY STATUS AND CHALLENGES FOR RESEARCH IN THE AMAZON.}

\begin{abstract}
Ecosystem services (SEs) have been understood as the benefits that human beings derive from ecosystems. Therefore, SE are directly and indirectly influenced by anthropic actions, which in the last decades have exerted strong pressure on them, placing them under threat of reduction or extinction. Some concepts, advances and advantages of the SEs approach are presented in this publication. The Amazon biome is very abundant in biodiversity and the provision of several SEs with impacts on different scales, but also many threats are suffered, and are also listed here. Finally, it is in this complex and diverse environmental and socioeconomic context, which is always very visible to national and international viewers, who present the great challenges of the research focused on the sustainability and provision of ecosystem services, being presented and discussed throughout the text.
\end{abstract}

Key words: orgtools for decision support, PES, biodiversity.

\footnotetext{
Bióloga, D. Sc. em Ciências da Engenharia Ambiental, pesquisadora da Embrapa Solos, Rio de Janeiro, RJ.

E-mail: rachel.prado@embrapa.br

(iD) https://orcid.org/0000-0002-1893-4915
} 


\section{Abordagem de serviços ecossistêmicos}

Segundo Hermann et al. (2011), o conceito de serviços ecossistêmicos remonta ao final dos anos 1960 e 1970. Contudo, a abordagem de SEs veio à tona a partir do Projeto Milênio. Solicitado pelo secretário-geral das Nações Unidas (ONU) Kofi Annan, em 2000, o projeto foi conduzido de 2001 a 2005, envolvendo mais de 1.300 cientistas e 95 países. Teve por objetivo avaliar as consequências que as mudanças nos ecossistemas trazem para o bem-estar humano e as bases científicas para subsidiar ações necessárias para melhorar a preservação e o uso sustentável desses ecossistemas (MILLENNIUM ECOSYSTEM ASSESSMENT, 2005; KUMAR, 2010).

Algumas variações no conceito de SEs têm sido encontradas na literatura, mas um dos conceitos mais aceitos e utilizados é o do próprio Projeto Milênio, que os define como os benefícios que o ser humano obtém dos ecossistemas (MILLENNIUM ECOSYSTEM ASSESSMENT, 2005). Alguns autores, bem como as políticas públicas, adotam o termo serviços ambientais. Este foi utilizado pela Organização das Nações Unidas para Alimentação e Agricultura (FAO), em seu relatório State of Food and Agriculture (FAO, 2007), como um "subconjunto de serviços ecossistêmicos que podem ser gerados como externalidades positivas de atividades humanas". Segundo o relatório isso ocorre, por exemplo, quando sistemas de produção agropecuária, além de gerar alimentos, fibras ou energia, contribuem para a manutenção da qualidade da água e do solo, para a beleza cênica ou a preservação de espécies. Na presente publicação, ambos os termos, serviços ecossistêmicos e serviços ambientais, serão utilizados como sinônimos.

De acordo com Andrade e Romeiro (2009), os serviços de suporte são aqueles essenciais para a produção dos outros serviços ecossistêmicos. Por exemplo: produção primária, formação e retenção de solo, ciclagem de nutrientes, ciclagem da água e provisão de habitat. Os serviços de provisão incluem os produtos que os ecossistemas oferecem, tais como alimentos e fibras, madeira, produtos medicinais e farmacêuticos, recursos ornamentais e água. Sua sustentabilidade não deve ser medida apenas em quantidade, e sim em qualidade e estado. Os serviços de regulação se relacionam às características regulatórias dos processos ecossistêmicos, regulação climática, controle de erosão, purificação de água, tratamento de resíduos, regulação de doenças humanas, polinização e proteção contra desastres. Os serviços culturais incluem a diversidade cultural, na medida em que a própria diversidade dos ecossistemas influencia a multiplicidade das culturas, valores religiosos e espirituais, geração de conhecimento (formal e tradicional), valores educacionais e estéticos.

A abordagem dos SEs tem algumas vantagens que podem ser destacadas: trabalho em múltiplas escalas, conexão entre a ciência e a política; ela ressalta, além dos aspectos ambientais, os sociais e econômicos relacionados ao bem-estar humano, visa promover a multifuncionalidade dos SEs, prevê compensação financeira ou não aos que atuam em prol dos serviços ecossistêmicos, entre outros aspectos. A partir de então o número de publicações relacionadas ao tema SE aumentou exponencialmente (Figura 1). 
Figura 1. Número de artigos indexados por ano na área de serviços ambientais/ecossistêmicos, destacando-se os hídricos.

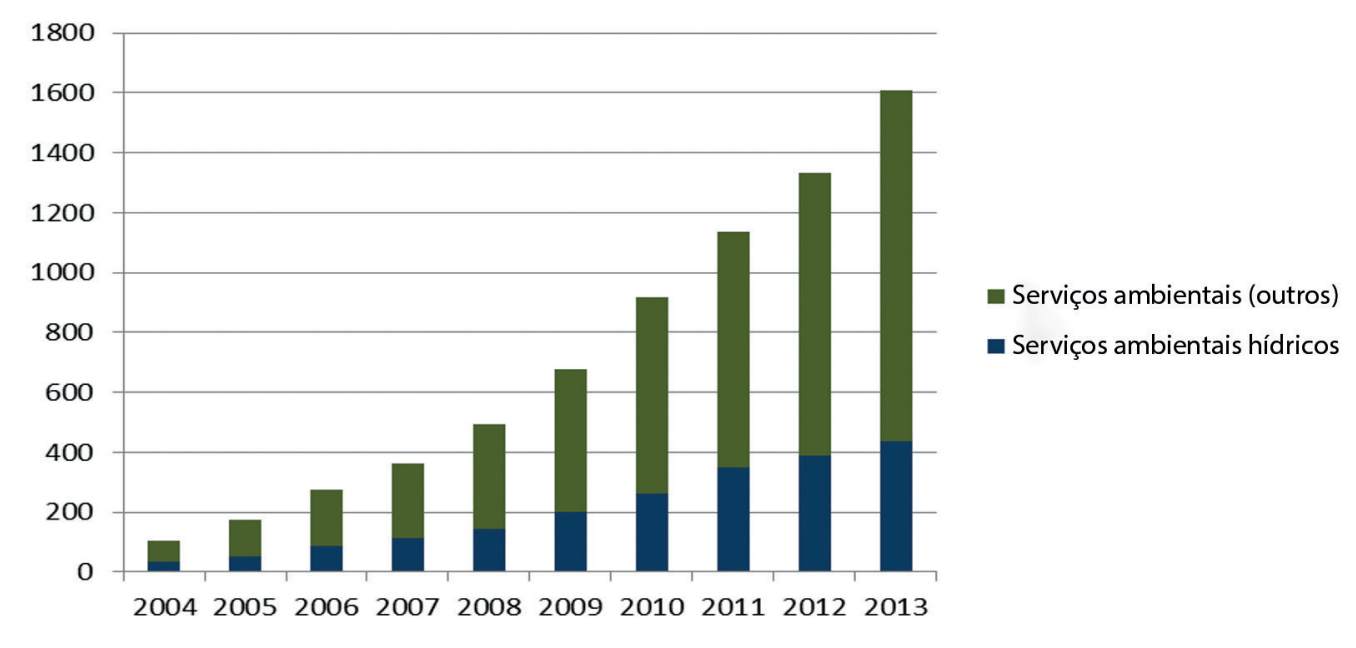

Fonte: Lima et al. (2015).

Existem diversas iniciativas globais visando promover pesquisas, desenvolvimento e políticas públicas voltadas à provisão de serviços ecossistêmicos, com destaque para: The Economics of Ecosystems and Biodiversity (TEEB), The Natural Capital Project, Intergovernmental Plataform on Biodiversity \& Ecosystem Services (IPBES), Ecosystem Services Partnership (ESP), Knowledge \& Learning Mechanism on Biodiversity \& Ecosystem Services (EKLIPSE), Europe Ecosystem Research Network (Alter-Net) e Water Funds.

No Brasil foram e continuam sendo realizados muitos estudos voltados à preservação da biodiversidade e à conservação ambiental nos diferentes biomas, devido à riqueza de biodiversidade e recursos naturais do País e aos processos de degradação por diferentes pressões antrópicas. Especificamente em relação ao tema SE, o número de publicações e o interesse também são crescentes. Mas destaca-se a atuação de gestores públicos, organizações não governamentais e políticas públicas, como tem sido nos pagamentos por serviços ambientais (PSAs).

Segundo Wunder et al. (2008), PSA é uma transação voluntária na qual um serviço ambiental bem definido ou um uso da terra que possa assegurar esse serviço é comprado por pelo menos um comprador de no mínimo um provedor sob a condição de que o provedor garanta a provisão desse serviço. Pagiola et al. (2012) apresentam diversas experiências de PSA no Brasil.

Para assegurar a geração e o provimento contínuo dos serviços ecossistêmicos nas áreas rurais, independentemente da escala, é preciso incluir nos custos de produção agropecuária os relacionados às práticas ambientais, necessárias à sustentabilidade do sistema de produção desenvolvido. Mas, principalmente para pequenos produtores rurais, arcar com os custos relacionados à preservação ambiental na sua propriedade torna-se inviável, visto que o percentual de lucro das atividades agropecuárias em sistema familiar de produção é ainda muito baixo. Especialmente nesses casos os PSAs se justificam (PRADO, 2014). 
Mas para que esse instrumento de compensação, dentre outros, possa ser efetivo na conservação ambiental e provisão de SE é preciso empenho maior de todos os setores da sociedade, com destaque para o governamental. São importantes a aprovação e implantação de uma política robusta de pagamento por serviços ambientais no País, maiores investimentos em recursos humanos e financeiros, além de apoio de resultados de pesquisa para suprir as lacunas de diversas naturezas encontradas até o momento. Neste contexto é preciso reconhecer e fortalecer o papel do produtor rural ou urbano como agente promotor da conservação. Por fim, o PSA é apenas um dos instrumentos de incentivo à provisão e manutenção dos SEs. Outros instrumentos e políticas públicas podem ser mais efetivos ou complementares às ações de um PSA, dependendo do contexto local.

\section{Serviços ecossistêmicos na Amazônia: abundância e ameaças}

\section{Abundância de serviços ecossistêmicos}

O bioma Amazônia ocupa uma área de $4.196 .943 \mathrm{~km}^{2}$, que corresponde a quase $50 \%$ do território nacional e é constituído principalmente por uma floresta tropical. A Amazônia é composta, no Brasil, pelos territórios do Acre, Amapá, Amazonas, Pará e de Roraima, e por parte do território do Maranhão, Mato Grosso, de Rondônia e Tocantins.

A região possui $27 \%$ de seu território protegido por unidades de conservação (UCs), e as terras indígenas (TI) na Amazônia Brasileira cobrem uma fração significativa da região, abrigando 173 etnias. Além de fundamentais para a reprodução física e sociocultural dos povos indígenas, as TIs, que do total de área demarcada do País 98\% é na Amazônia, são áreas importantes para a conservação da biodiversidade regional e global. Apesar desses evidentes benefícios prestados pelas TIs para o meio ambiente amazônico, o papel delas para a mitigação da mudança do clima e equilíbrio climático da região ainda é pouco reconhecido, mas destaca-se que as florestas sob a guarda dos povos indígenas na Amazônia Brasileira representam um imenso armazém de carbono (aproximadamente 13 bilhões de toneladas) (CRISOSTOMO et al., 2015). A Figura 2 apresenta as UCs apoiadas pelo Programa Áreas Protegidas da Amazônia (Arpa), TIs e demais áreas protegidas na Amazônia.

A quantidade de serviços ecossistêmicos gerados nesse bioma é uma das maiores do mundo. Fearnside (2008) agrupa os serviços ecossistêmicos da Amazônia em: biodiversidade, água e mitigação do aquecimento global.

Em termos de biodiversidade, alguns grupos de organismos - como aves, peixes de água doce, borboletas e primatas - são extremamente diversos na Amazônia. Nenhum outro domínio no mundo apresenta tantas espécies desses grupos. A riqueza biológica da Amazônia é tão grande que incorpora, total ou parcialmente, elementos de 49 das 200 ecorregiões mundiais. A magnitude da diversidade da Amazônia é imensa, sendo a região constituída por mais de 600 diferentes tipos de habitats terrestres e de água doce. Com toda essa diversidade de ambientes e extensão, a Amazônia ainda é uma fronteira do conhecimento, com espécies novas sendo descobertas a cada ano. Entre 1999 e 2009, por exemplo, mais de 1.200 espécies foram descobertas por cientistas na região. Entre elas estão 639 plantas, 257 peixes, 216 anfíbios, 55 répteis, 39 mamíferos e 16 
aves (BRASIL, 2010). Cerca de 10\% de toda a diversidade do planeta encontra-se na região, inclusive muitas espécies ameaçadas de extinção e também espécies que ocorrem exclusivamente na Amazônia.

Figura 2. Unidades de conservação apoiadas pelo Programa Áreas Protegidas da Amazônia (Arpa), terras indígenas (TIs) e demais áreas protegidas na Amazônia.

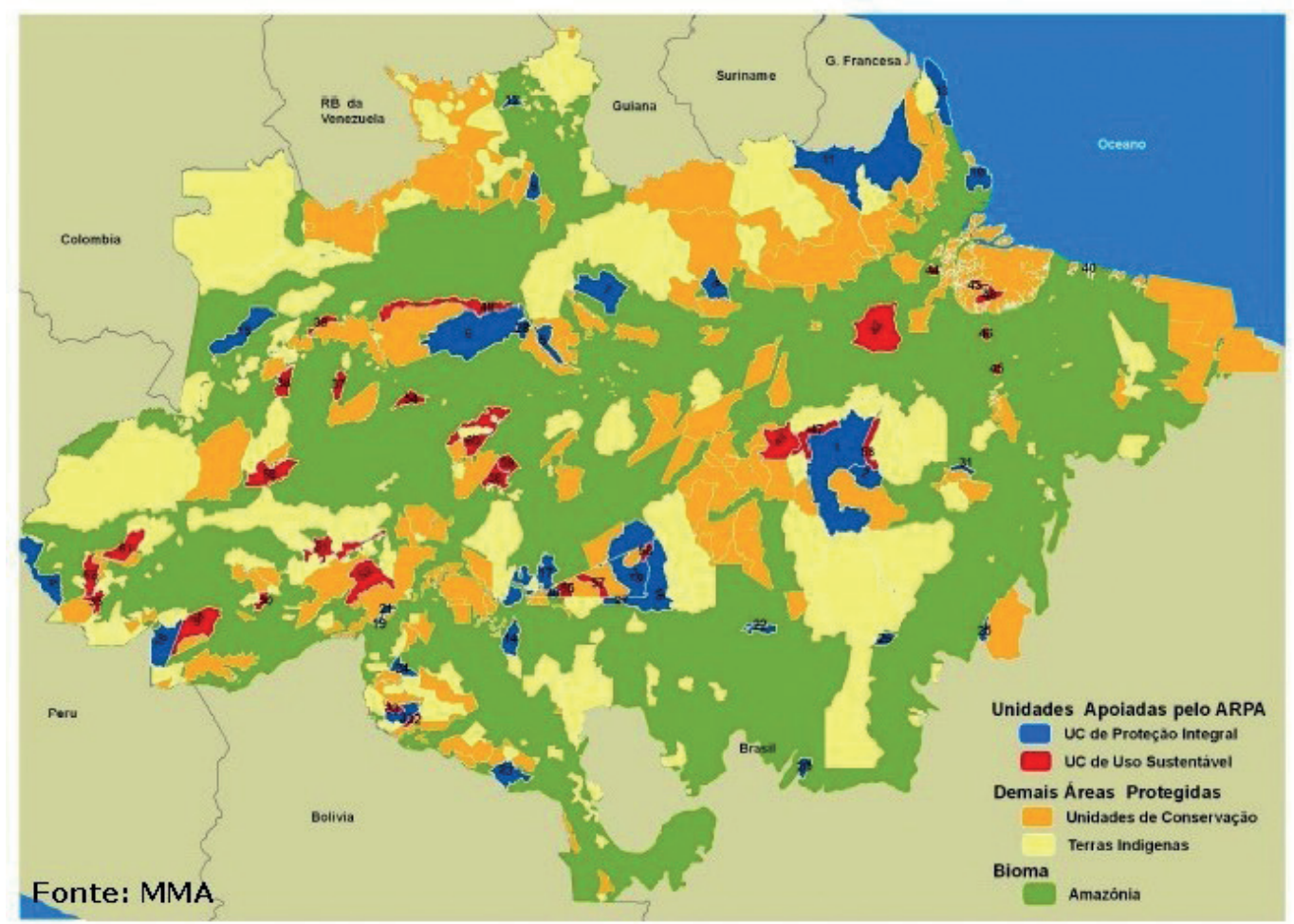

Fonte: Brasil (2012).

Um inventário realizado por Brasil (2012) em 39 das 62 UCs apoiadas pelo Arpa identificou elevado número de espécies encontradas (Figura 3).

Figura 3. Número de espécies encontradas em 39 unidades de conservação (UCs) da Amazônia.

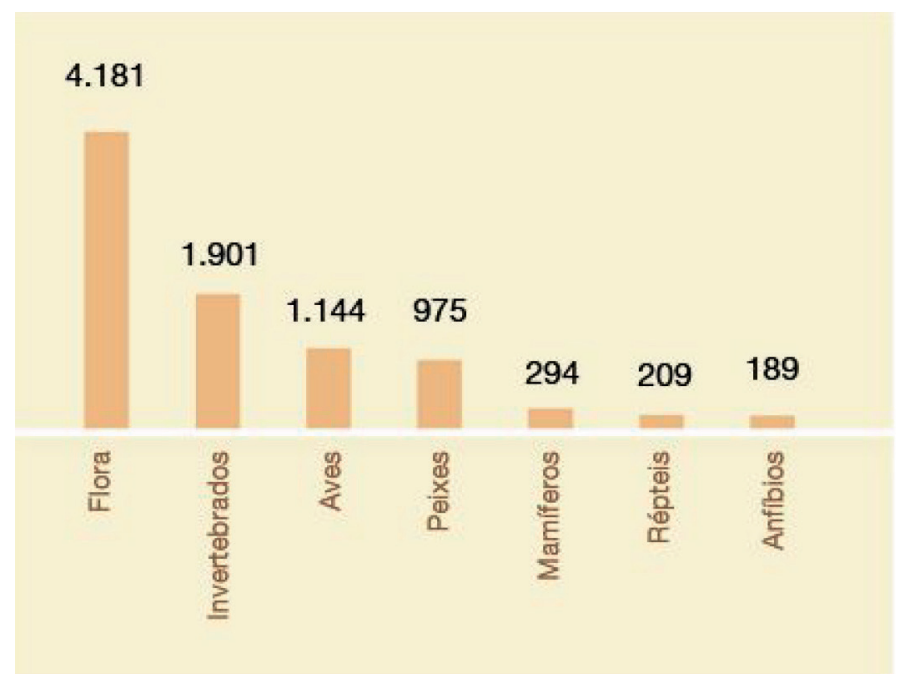

Fonte: Brasil (2012). 
A biodiversidade da Amazônia ainda é muito pouco conhecida pelo homem. Descobrir, estudar e proteger esse patrimônio natural, que pode conter inúmeros benefícios para a sociedade, é uma missão fundamental e de interesse ambiental, social e econômico. O estado do Amazonas detém $92,84 \%$ de sua cobertura florestal preservada.

Em relação à água, a Bacia Amazônica é a maior bacia hidrográfica do Brasil e do mundo, abrangendo uma área de 7 milhões de $\mathrm{km}^{2}$, compreendendo terras de vários países da América do Sul (Peru, Colômbia, Equador, Venezuela, Guiana, Bolívia e Brasil). O rio principal da bacia, o Amazonas, é o maior do mundo, nascendo na Cordilheira dos Andes (Peru), e quando entra no Brasil é intitulado de Rio Solimões. A confluência do Rio Solimões com o Rio Negro constitui o Rio Amazonas. Dentro da bacia em questão é identificado um enorme potencial para geração de energia elétrica, uma vez que muitos afluentes do Rio Amazonas possuem características que se adéquam às condições básicas para a construção de usinas hidrelétricas. Outra potencialidade encontrada na Bacia Amazônica é a viabilidade para o transporte fluvial, tendo em vista que a topografia é plana e a maioria dos rios são caudalosos, sendo o principal meio de deslocamento e de comunicação da região Norte do Brasil.

\section{Ameaças aos serviços ecossistêmicos}

Apesar da imensa biodiversidade do bioma Amazônia, estima-se a perda de ambientes naturais entre 15\% e 18\% (FERREIRA et al., 2012). Somada às perdas na biodiversidade, pode-se mencionar a degradação das terras e da água como consequência de diversas pressões antrópicas e mudanças climáticas, com reflexos diretos e indiretos na provisão dos SEs.

O Instituto Socioambiental (ISA) (2015) realizou estudo mapeando e classificando as principais pressões e ameaças às UCs na Amazônia Brasileira. Dentre elas destacam-se as mineradoras, hidrelétricas, ferrovias/rodovias e os empreendimentos relacionados à exploração de petróleo e gás. Além do monitoramento do desmatamento da Amazônia pelo Programa de Cálculo do Desflorestamento da Amazônia (Prodes), o Instituto do Homem e Meio Ambiente da Amazônia (Imazon) detectou que a perda da floresta dentro de UCs, em relação ao desmate total da Amazônia Legal, dobrou entre 2012 e 2015, passando de 6\% para 12\%. Ferreira et al. (2005) apresentam a importância das áreas protegidas para a conservação da biodiversidade. O desmatamento tem ocorrido principalmente pela dinâmica de uso da terra em função da ampliação das fronteiras agropecuárias, além da exploração madeireira. Barlow et al. (2016) discorrem sobre os impactos antropogênicos nas florestas tropicais sobre a perda de biodiversidade.

Especificamente no estado do Amazonas, segundo IBGE (2017), a agropecuária cresceu $21 \%$ nos últimos anos. Para Carrero et al. (2015), o rebanho bovino nesse estado cresceu 73\% entre 1990 e 2012, índice superior à média nacional, que foi de 24\% no mesmo período. Segundo o Tribunal de Contas da União (TCU) (2015), mais de 90\% dos estabelecimentos rurais são familiares, com sérios problemas de regularização fundiária, e mais de $80 \%$ dos alimentos consumidos no Amazonas vêm de outros estados. Também se destaca o Índice de Desenvolvimento Humano Municipal (IDHM), que na região amazônica, em 2010, predominavam as classes: baixo e muito baixo (Figura 4). Esses dados reforçam a fragilidade dos sistemas socioecológicos da região amazônica com reflexos negativos nos serviços ecossistêmicos. 
Figura 4. Índice de Desenvolvimento Humano Municipal (IDHM) em 2010 da Amazônia.

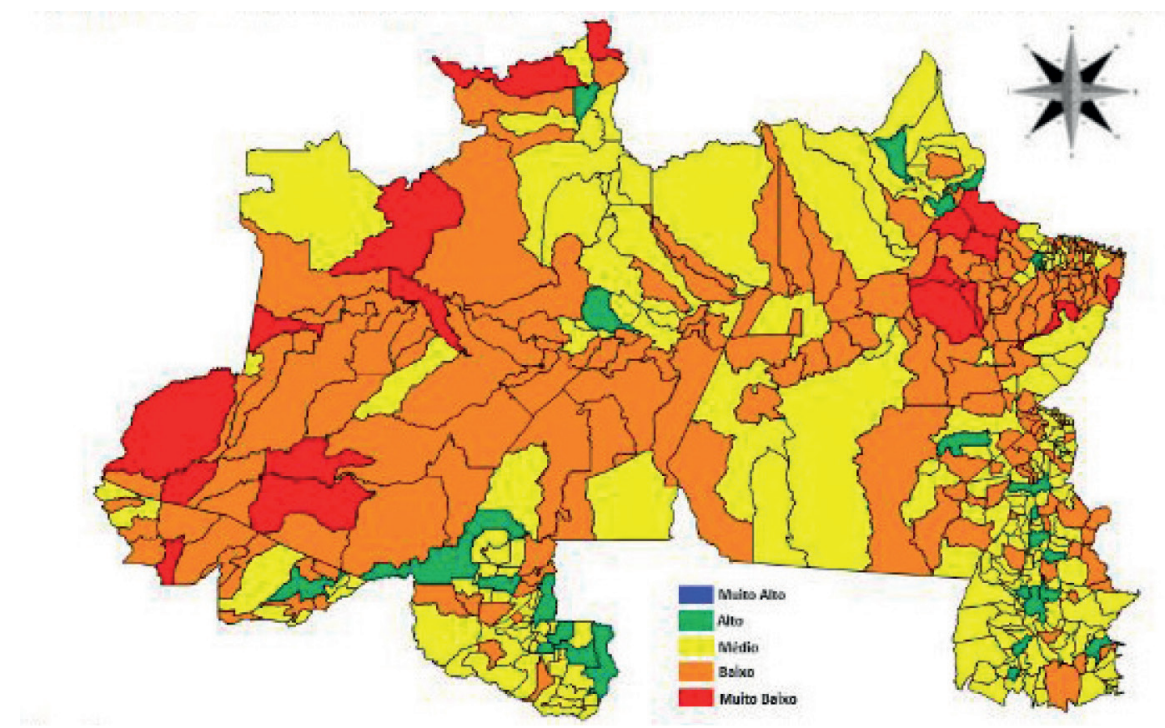

Fonte: Coordenação-Geral de Estudos Econômicos e Coordenação de Empresas $(\mathrm{Cogec})$, usando Software IpeaGeo 2.0.0.

As águas da Bacia Amazônica historicamente têm sofrido com os impactos negativos da construção de grandes usinas hidrelétricas (JUNK; MELLO, 1990), dos esgotos sanitários das grandes cidades, bem como com a sedimentação dos rios por processos erosivos advindos do desmatamento e uso inadequado do solo na agropecuária.

Como as mudanças climáticas estão associadas à emissão de gases de efeito estufa (GEE) e às mudanças de uso e cobertura da terra (Figura 5), o que está bastante presente na Amazônia por causa do desmatamento e expansão da agropecuária, essa região tem sentido os seus impactos, como, por exemplo, no seu regime hídrico. Sorribas et al. (2016) mencionam que eventos hidrológicos ocorridos nas últimas décadas, como as enchentes em 2009, 2012 e 2014 e as secas em 2005 e 2010, impactaram a região, alertando cientistas, governos e o público em geral sobre os impactos na variabilidade climática.

Figura 5. Emissões brutas de GEE no Brasil de 1990-2016 (tCO2e-GWP AR5).

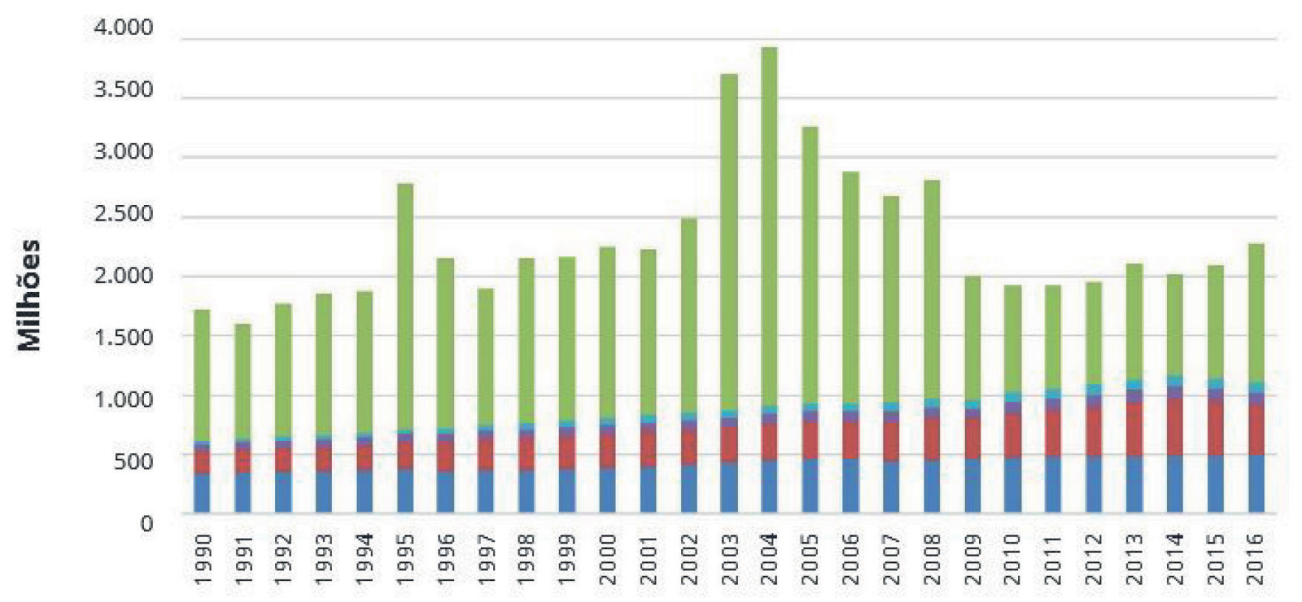

Agropecuária —Energia — Processos Industriais — Resíduos — Mudanças de Uso da Terra

Fonte: Observatório do Clima (2019). 
As condições limnológicas e ecológicas em lagos de várzeas e zonas úmidas estão intimamente associadas à dinâmica de inundação. Os rios da Amazônia e as áreas alagadas produzem grandes quantidades de $\mathrm{CO} 2$ e metano, que são significativos no ciclo de carbono regional. Além disso, a dinâmica de inundação influencia a estrutura da vegetação, o transporte de sedimentos, a distribuição de peixes e o rendimento pesqueiro. Mudanças futuras no clima e na hidrologia provavelmente alterarão a inundação da planície aluvial e as condições ecológicas desses ecossistemas (SORRIBAS et al., 2016).

\section{Serviços ecossistêmicos: desafios para a pesquisa na Amazônia}

Estudo realizado pelo Tribunal de Contas da União (2015) identificou alguns desafios locais para o desenvolvimento do estado do Amazonas, sendo eles: implementar a concepção de desenvolvimento sustentável e redução do desmatamento; reduzir as desigualdades sociais, visto que parcela significativa da população do estado vive em condições insalubres e abaixo da linha da pobreza; aumentar a contribuição do Amazonas para a formação do Produto Interno Bruto do Brasil, pois não alcançava 2\%; e compatibilizar o desenvolvimento econômico, social e ambiental. Esses desafios são imensos e seguramente são comuns para todos os estados da Amazônia, com resultados de pesquisa que poderão contribuir muito para sua superação.

Pela própria dimensão da Amazônia já são esperadas maiores dificuldades que em outros biomas para o desenvolvimento da pesquisa voltada à avaliação, valoração e provisão de SE, pois as distâncias são muito grandes, as florestas e os rios são abundantes e os povos se encontram dispersos, implicando também maiores custos de logística. Desta forma, os recursos financeiros e humanos destinados à pesquisa na Amazônia precisam ser assegurados e ter continuidade. Outros desafios mais inerentes à pesquisa são listados na sequência.

Muitos dados e informações têm sido gerados na Amazônia ao longo dos últimos tempos, com atuação de instituições nacionais e internacionais, que têm investido recursos humanos e financeiros para o conhecimento e a quantificação dos recursos naturais nela presentes, bem como para dimensionar as ameaças que ela sofre e suas potencialidades, com destaque para o Instituto de Pesquisa Ambiental da Amazônia (Ipam).

Contudo, um desafio é conectar e integrar diferentes grupos de pesquisa, bases de dados e infraestrutura para que realmente possam servir de suporte à tomada de decisão em prol da sustentabilidade e provisão de SE. Projetos integradores e redes de pesquisa têm muito a contribuir nesse sentido, gerando ferramentas para a seleção de áreas prioritárias à conservação, organização e disponibilização de bases de dados sobre a biodiversidade e práticas de manejo adequadas da terra, bem como sobre o monitoramento dos SEs em ambientes naturais e antropizados. Também são importantes a geração e validação de tecnologias sociais que permitam melhorar a qualidade de vida das comunidades que vivem dos produtos da floresta e da agricultura, permitindo a redução das pressões sobre os SEs. Nesse contexto é necessário estabelecer parcerias entre órgãos do governo, de fomento, bancos, associações de produtores, assistência técnica, universidades, institutos de pesquisa e iniciativa privada. 
De modo geral, os equipamentos para coleta e métodos de análise dos dados relacionados aos SEs possuem custo bastante elevado. Em grandes programas, projetos e iniciativas voltados à conservação, desenvolvidos em grandes áreas, é preciso avançar com o desenvolvimento e a utilização de métodos simplificados e de baixo custo, abrangendo inclusive os atores locais e a população envolvida, para que possam se apropriar e participar de avaliações e monitoramento dos SEs. Desta forma, aumenta-se a percepção dos envolvidos e afetados sobre a importância do manejo adequado das terras e da manutenção dos SEs e permite-se continuidade do monitoramento, visto que os projetos são de curta duração.

O termo "multifuncionalidade da agropecuária" tem sido utilizado com vários significados no debate sobre políticas agropecuárias, dependendo do país e do contexto em que surgiu (ORGANISATION FOR ECONOMIC COOPERATION AND DEVELOPMENT, 2001). No Brasil, o uso dessa abordagem ainda é limitado, mas tem ganhado força quando se demonstra o potencial da agropecuária em prover $\mathrm{SE}$, além da sua função primária de produtora de alimentos, fibra e energia. Esse conceito se traduz numa abordagem de provisão de SE pelos agroecossistemas.

Ferreira et al. (2012) destacam que a avaliação e o manejo dos SEs em paisagens rurais com a presença da agropecuária aparecem no topo das prioridades por diversas razões. Primeiro que áreas agropecuárias estão entre os ambientes mais extensos da superfície terrestre; segundo que o aumento da produção de alimentos e fibras tem sido alcançado a custo da degradação de outros SEs.

A prestação de SE pelos ambientes naturais é fundamental para a produção agropecuária e está altamente correlacionada à estrutura da paisagem em que a agropecuária esteja embutida. Paisagens diversificadas, isto é, onde o componente arbóreo esteja presente nas APPs, em reserva legal e as culturas sejam diversificadas e estejam sob manejo adequado do solo e da água, como, por exemplo, os sistemas agroecológicos, agroflorestais e de integração lavoura-pecuária-floresta, tendem a apresentar maior multifuncionalidade em relação aos SEs. São, portanto, capazes de se manter em equilíbrio para a regulação do clima, das enchentes, da erosão, da ciclagem de nutrientes, da água, permitindo a polinização, a permeabilidade da fauna e a provisão de alimentos mais saudáveis a partir da redução da utilização de insumos. Outro aspecto positivo é que são capazes de atrair a atenção do turismo e agregar valor aos produtos delas advindos.

Desta forma, é um desafio importante a se considerar o desenvolvimento de pesquisas capazes de avaliar e valorar os benefícios da multifuncionalidade da paisagem, visando atrair a atenção de investidores e governos para esse tipo de prática. Quando se pretende compreender o grau de harmonia e interação entre os SEs de uma região, uma tendência internacional é analisar os conflitos (trade-offs) e sinergias entre eles. Há diversos métodos disponíveis na literatura, tais como Power (2010) e Turkelboom et al. (2016).

Outro grande desafio à pesquisa visando à provisão de $\mathrm{SE}$ é gerar subsídios à certificação da produção sustentável e agregação de renda aos sistemas produtivos que fazem uso sustentável da biodiversidade. Isso significa gerar indicadores e métodos capazes de medir o impacto desses sistemas de produção nos SEs e benefícios às comunidades envolvidas. Essas ferramentas poderão certificar a produção sustentável visando à abertura de novos mercados e possibilitando 
novas oportunidades às comunidades tradicionais e marginalizadas da Amazônia, promovendo a sua inclusão no mercado e fazendo a ponte entre o rural e o urbano.

Por fim, é preciso que os pesquisadores gerem material, publicações e outras formas de disseminar o conhecimento em linguagem acessível, visando ao apoio às políticas públicas. $\mathrm{O}$ conhecimento gerado na academia precisa atingir a sociedade e ser apropriado por ela, gerando soluções práticas aos grandes problemas agroambientais que afligem a região amazônica.

\section{Considerações Finais}

- Os conceitos relacionados à abordagem de serviços ecossistêmicos estão bem definidos, e muitas são as ferramentas e métodos que podem ser utilizados, mas é preciso internalizá-los e colocá-los em prática.

- Os mecanismos de PSA devem continuar sendo desenvolvidos, ampliados e aperfeiçoados com o apoio de ferramentas de pesquisa, mas sobretudo por meio de políticas públicas efetivas.

- Apesar da abundância de biodiversidade, muitas são as ameaças sobre o bioma Amazônia no que tange aos serviços ecossistêmicos, destacando-se o avanço do desmatamento, a ocupação de terras e a construção de hidrelétricas com visão conservacionista limitada, sem contar os impactos das mudanças climáticas.

- Portanto, muitos são os desafios para a pesquisa em prol dos SEs na Amazônia, podendo ser resumidos em: integração de diferentes grupos de pesquisa, bases de dados e infraestrutura; desenvolvimento e utilização de métodos simplificados e de baixo custo; avaliação e valoração dos benefícios da multifuncionalidade da paisagem; geração de subsídios à certificação da produção sustentável e agregação de renda aos sistemas produtivos que fazem uso sustentável da biodiversidade, com maior inclusão dos povos tradicionais e marginalizados.

\section{Referências}

ANDRADE, D. C.; ROMEIRO, A. R. Serviços ecossistêmicos e sua importância para o sistema econômico e o bem-estar humano. Texto para Discussão (IE/UNICAMP), n. 155, p. 45, 2009.

BARLOW, J.; LENNOX, G. D.; FERREIRA, J.; BERENGUER, E.; LEES, A. C.; MAC NALLY, R.; PARRY, L.; OLIVEIRA-JUNIOR, R. C.; VENTURIERI, A.; GARDNER, T. Anthropogenic disturbance in tropical forests can double biodiversity loss from deforestation. Nature, v. 535, n. 7610, p. 144-147, 2016.

BRASIL. Ministério do Meio Ambiente. ARPA Biodiversidade. 2012. Disponível em: http://arpa.mma. gov.br/wp-content/uploads/2012/10/arpaBiodiversidade.pdf. Acesso em: 08 mar. 2019.

CARRERO, G. C.; ALBUJA, G.; FRIZO, P.; HOFFMAN, E. K.; ALVES, C.; BEZERRA, C. S. A cadeia produtiva da carne bovina no Amazonas. Manaus: IDESAM: WWF: APUÍ, 2015. 41 p. Disponível em: http://www.idesam.org.br/publicacao/cadeia-produtiva-corte-amazonas.pdf. Acesso em: 08 mar. 2019. 
CRISOSTOMO, A. C.; ALENCAR, A.; MESQUITA, I.; SILVA, I. C.; DOURADO, M. F.; MOUTINHO, P.; CONSTANTINO, P. A.; PIONTEKOWSKI, V. Terras indígenas na Amazônia brasileira: reservas de carbono e barreiras ao desmatamento. Brasília, DF: IPAM, 2015. 12 p.

FAO. Food and Agriculture Organization. State of Food and Agriculture, 2007.

FEARNSIDE, P. M. Manutenção da Floresta Amazônica como fonte de serviços ambientais. Anais da Academia Brasileira de Ciências, v. 80, n. 1, mar. 2008. Doi: http://dx.doi.org/10.1590/ S0001-37652008000100006.

FERREIRA, L.; VENTICINQUE, E.; ALMEIDA, S. O desmatamento na Amazônia e a importância das áreas protegidas. Estudos Avançados, v. 19, n. 53, p. 157-166, 2005.

FERREIRA, J.; PARDINI, R.; METZGER, J. P.; FONSECA, C. R.; POMPEU, P. S.; SPAROVEK, G.; LOUZADA, J. Towards environmentally sustainable agriculture in Brazil: challenges and opportunities for applied ecological research. Journal of Applied Ecology, v. 49, p. 535-541, 2012.

HERMANN, A.; SCHLEIFER, S.; WRBK, T. O conceito de serviços ecossistêmicos em relação à pesquisa da paisagem: uma revisão. Living Rev. Landscape Research, v. 5, p. 1-37, 2011.

INSTITUTO BRASILEIRO DE GEOGRAFIA E ESTATÍSTICA - IBGE. Censo Agropecuário 2017. Rio de Janeiro, 2017.

INSTITUTO SOCIOAMBIENTAL - ISA. Unidades de conservação da Amazônia brasileira: ameaças e pressões. 2015. Disponível em: https://www.socioambiental.org/pt-br/mapas/unidades-de-conservacao-na-amazonia-brasileira-pressoes-e-ameacas-2015. Acesso em: 08 mar. 2019.

JUNK, W. J.; MELLO, J. A. S. N. Impactos ecológicos das represas hidrelétricas na bacia amazônica brasileira. Estudos Avançados, v. 4, n. 8, p. 126-143, 1990.

KUMAR, P. (Ed.). The economics of ecosystems and biodiversity: Ecological and Economic Foundations (TEEB). London: Earthscan, 2010. 410 p.

LIMA, R. A.; PEDREIRA, B. C. C. G.; CLEMENTE, E. P.; PRADO, R. B. O avanço do conhecimento na área de serviços ambientais. In: CONGRESO INTERNACIONAL DE SERVICIOS ECOSISTÉMICOS EN LOS NEOTRÓPICOS, 4., 2015, Mar del Plata. Da pesquisa à ação: livro de resumos. Mar del Plata: GEAP, 2015.

MILLENNIUM ECOSYSTEM ASSESSMENT - MEA. Ecossistemas e bem-estar humano: síntese. Washington, DC: Island Press, 2005.

OBSERVATÓRIO do Clima. Disponível em: http://www.observatoriodoclima.eco.br/. Acesso em: 08 mar. 2019.

ORGANISATION FOR ECONOMIC COOPERATION AND DEVELOPMENT - OECD. Multifunctionality: towards an analytical framework. Paris: OECD Publishing, 2001. 160 p.

PAGIOLA, S.; GLEHN, H. C. von; TAFFARELLO, D. Experiências do Brasil em pagamentos por serviços ambientais. In: SÃO PAULO (Estado). Secretaria do Meio Ambiente. Coordenadoria de Biodiversidade e Recursos Naturais. Experiências de pagamentos por serviços ambientais no Brasil. São Paulo, 2012.

POWER, A. G. Serviços ecossistêmicos e agricultura: compensações e sinergias. Philosophical Transactions of the Royal Society B-Biological Sciences, v. 365, p. 2959-2971, 2010.

PRADO, R. B. Serviços ecossistêmicos e ambientais na agropecuária. In: PALHARES, J. C. P.; GEBLER, L. (Ed.). Gestão ambiental na agropecuária. Brasília, DF: Embrapa, 2014. p. 414-456. 
PRADO, R. B.; INÁCIO, M.; LIMA, A. P. M.; SCHULER, A. E.; GUIMARÃES, J.; FIDALGO, E. C. C.; TURETTA, A. P. D.; MONTEIRO, J. M; MARTINS, A. L.; OLIVEIRA, A. P.; CLEMENTE, E. P; PEDREIRA, B. C. C. G. Evolução das iniciativas de pagamentos por serviços ambientais hídricos no Brasil. Cadernos de Ciência \& Tecnologia, v. 36, p. 1-13, 2019.

SORRIBAS, M. V.; PAIVA, R. C. D.; MELACK, J. M.; BRAVO, J. M.; CARVALHO, L.; BEIGHLEY, E.; FORSBERG, B.; COSTA, M. H. Projeções dos efeitos das mudanças climáticas na descarga e inundação na bacia amazônica. Climatic Change, v. 136, n. 3-4, p. 555-570, 2016.

TRIBUNAL DE CONTAS DA UNIÃO - TCU. Relatório do levantamento de auditoria realizado pela Secretaria de Controle Externo do Estado do Amazonas. 2015. Disponível em: https://portal.tcu. gov.br/data/files/EE/A0/48/44/43F935108BFDC6351A2818A8/013.329\%20Fisc\%20Nordeste\%20-\%20 Regi_o\%20Norte.pdf. Acesso em: 08 mar. 2019.

TURKELBOOM, F.; THOONEN, M.; JACOBS, S.; GARCÍA-LLORENTE, M.; MARTÍN-LÓPEZ, B.; BERRY, P. Trocas de serviços ecossistêmicos e sinergias. In: POTSCHIN, M.; JAX, K. (Ed.). Livro de referência dos serviços ecossistêmicos OpenNESS. EC FP7 Grant Agreement, n. 30, p. 8428, 2016.

WUNDER, S.; BORNER, J.; TITO, M. R.; PEREIRA, L. Pagamentos por serviços ambientais: perspectivas para a Amazônia Legal. Brasília, DF: Ministério do Meio Ambiente, 2008.

WWF-BRASIL. Amazônia viva! Uma década de descoberta 1999-2009. Brasília, DF, 2010. 57 p. 